\title{
Blocking or enhancing effects of some basic emollients in UVA penetration*
}

\author{
Sevgi Akarsu ${ }^{1}$ \\ Ozlem Ozbagcivan ${ }^{1}$
}

\author{
Emel Fetil ${ }^{1}$ \\ Ali Tahsin Gunes ${ }^{1}$
}

DOI: http://dx.doi.org/10.1590/abd1806-4841.20186869

\begin{abstract}
BACKGROUND: Topical agents used in combination with phototherapy or photochemotherapy may have both blocking or enhancing effects in ultraviolet rays.

Oвjестіve: In this in vivo study, the effects of topical petrolatum, basis cream, glycerine, and olive oil on the transmission of ultraviolet A radiation were investigated.

MetHODS: A test was performed to determine the minimal phototoxic dose on 29 volunteers with only psoralen plus ultraviolet A (PUVA) and then the same test was repeated with white petrolatum, basis cream, glycerine, olive oil, and sunscreen $(0.3 \mathrm{cc} / 25 \mathrm{~cm} 2)$. The effects of each agent on the minimal phototoxic dose were determined after $72 \mathrm{~h}$.

RESUlTS: When compared to pure PUVA, there was a statistically significant increase in the mean minimal phototoxic dose values by the application of white petrolatum $(\mathrm{P}=0.011)$, but there was no significant increase or decrease in the mean minimal phototoxic dose values after the application of basis cream $(\mathrm{P}=0.326)$, glycerine $(\mathrm{P}=0.611)$ or olive oil $(\mathrm{P}=0.799)$.

STUDY LIMITATIONS: Low number of patients

CONCLUSION: The application of white petrolatum, which has a blocking effect, and also of basis cream immediately before PUVA therapy should not be recommended. Although we specify that glycerine and maybe olive oil can be used before photochemotherapy, there is a need for further research in larger series.
\end{abstract}

Keywords: Petrolatum; PUVA therapy; Skin cream; Ultraviolet rays

\section{INTRODUCTION}

Various topical agents may be used with ultraviolet B (UVB) and psoralen plus ultraviolet A (PUVA) therapy. Even though emollients are usually the constituents of the phototherapeutic regimen and they are frequently used as skin-care products to prevent the drying effect of UVB radiation, we still have no certain evidence for selecting the optimal emollient to be used in combination with PUVA photochemotherapy. However, the adequate penetration of UVA radiation into the deeper portions of skin is very important to achieve an effective treatment while using emollients with PUVA regimens. ${ }^{1-7}$

Certain emollients seem to have significant photoprotective properties by diminishing the UV penetration; however, the transmission of radiation has been found to be increased following the application of some particular lipophilic compounds. ${ }^{1-10}$ So, according to their effects, topical administration of emollients immediately before PUVA therapy can be recommended if they are known to enhance UVA transmission, or can be avoided if they are known to block UVA transmission. Therefore, we should know about the blocking or enhancing effects of the frequently used emollients on the UVA penetration. In this in vivo study, we examined the effects of some basic emollients (petrolatum, basis cream, glycerine, olive oil) on the transmission of UVA.

\section{METHODS}

The study was designed as a single-blind, vehicle-controlled study to determine the minimal phototoxic dose (MPD) after the application of white petrolatum, basis cream, glycerine, and olive oil. The test was carried out on 29 volunteers with the diagnosis of psoriasis, lichen planus, parapsoriasis, mycosis fungoides, and vitiligo before their PUVA therapy. Eleven patients had skin type II, 15 patients had skin type III, and three patients had skin type IV.

\footnotetext{
Received on 02.01.2017.

Approved by the Advisory Board and accepted for publication on 14.02.2017.

* Work was performed at the Department of Dermatology, Faculty of Medicine - Dokuz Eylul University Izmir, Turkey.

Financial support: None.

Conflict of interest: None

1 Department of Dermatology, Faculty of Medicine, Dokuz Eylul University - Izmir, Turkey.
}

E-mail: ozlem.ozbagcivan@deu.edu.tr 
The study was approved by an ethics committee. All participants were informed and their consent forms were obtained. The patients were untanned and not taking any medication. Waldmann $8001 \mathrm{~K}$ (Waldmann Lichttechnik GmbH, Schwenningen, Germany) cabinet was used for the light source of UVA.

The MPD test was performed on the volunteers 2 hours after taking $0.6-0.8 \mathrm{mg} / \mathrm{kg}$ 8-methoxypsoralen. According to the skin types of the volunteers, the doses of UVA were determined. Patients with skin types I and II were exposed to the doses of $0.5-5 \mathrm{~J} / \mathrm{cm}^{2}$ and patients with skin types III and IV were exposed to the doses of 1.5$9 \mathrm{~J} / \mathrm{cm}^{2}$ of UVA. ${ }^{[7]}$ The MPD values were determined by irradiating the $4 \mathrm{~cm}^{2}$ of uninvolved skin on the back of each patient in eight different areas, in one row, with the increments of $1 \mathrm{~J} / \mathrm{cm}^{2}(0.5 \mathrm{~J} /$ $\mathrm{cm}^{2}$ only for the first one). In addition, 5 parallel rows of skin were tested after the application of different topical agents. The first row was only irradiated by UVA without applying any topical agent to determine the MPD. Then white petrolatum, basis cream, glycerine, olive oil, and a sunscreen factor 30 (titanium dioxide+triethanolamine) were applied in a layer $\left(0.3 \mathrm{cc} / 25 \mathrm{~cm}^{2}\right)$ respectively to the adjacent parallel rows and irradiated with UVA, immediately after the application of the agents.

The results were evaluated after 72 hours by a blinded investigator and the MPD values were determined for each row (Figure 1). Wilcoxon signed ranks test and paired t-tests were used to compare the results obtained after the application of different emollients with each other and pure PUVA. $p<0.05$ was considered as statistically significant.

\section{RESULTS}

The results of the MPD tests of the patients with pure PUVA and with the applications of white petrolatum, basis cream, glycerine, and olive oil are shown in table 1 . Sunscreen caused a total blocking effect on all subjects.

When compared with pure PUVA, the higher mean MPD values were detected with white petrolatum followed by the basis cream. There was a very slight increase in the MPD with olive oil, whereas there was a slight decrease with glycerine. Although the

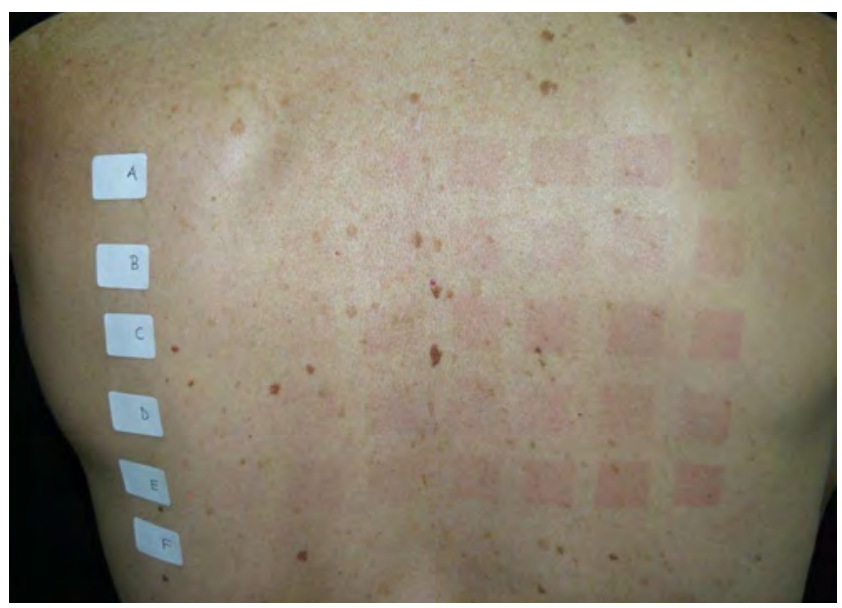

FiguRE 1: A patient seen $72 \mathrm{~h}$ after MPD test (A, psoralen plus UVA; B, petrolatum; C, glycerine; D, basis cream; E, olive oil; F, sunscreen)
MPD values detected after the application of basis cream, glycerine, and olive oil did not show statistically significant difference compared with pure PUVA, the mean MPD value detected after the application of white petrolatum showed a statistically significant difference. These findings showed that the MPD values were significantly increased by the application of white petrolatum, but there was no significant increase or decrease in the MPD values after the application of basis cream, glycerine or olive oil.

The MPD values detected after the application of white petrolatum were compared, one by one, with the MPD values detected after the application of basis cream, olive oil, glycerine and each comparison was found statistically significant. Additionally, the MPD values detected after the application of basis cream were compared with the MPD values detected after the application of glycerine and a statistically significant difference was found. However, there was no statistically significant difference between the MPD values after the application of olive oil when compared with basis cream and glycerine. The results of the Wilcoxon signed ranks test of all paired groups are shown in table 2 .

\section{DISCUSSION}

Phototherapy and photochemotherapy may be used in combination with various topical agents in the treatment of several skin diseases. At this point, the interference of these agents with UVB or UVA is an important issue whether it may be of the blocking or

TABLE 1: The mean MPD scores for pure PUVA and test substances

\begin{tabular}{lll}
\multirow{2}{*}{ Preparation } & \multicolumn{2}{l}{ MPD $(\mathrm{J} / \mathrm{cm} 2)$} \\
\cline { 2 - 3 } & Range & Mean \pm SD \\
\hline Pure PUVA & $1.00-7.00$ & $3.86 \pm 1.68$ \\
Petrolatum & $1.00-9.00$ & $4.45 \pm 2.06$ \\
Basis cream & $1.00-8.00$ & $4.07 \pm 1.83$ \\
Glycerine & $0.50-7.00$ & $3.79 \pm 1.89$ \\
Olive oil & $0.50-8.00$ & $3.89 \pm 1.92$ \\
\hline
\end{tabular}

SD, standard deviation; PUVA, psoralen plus ultraviolet A; MPD, minimal phototoxic dose.

TABLE 2: Comparison of the effects of emollients with each other and pure PUVA

\begin{tabular}{lc} 
Paired group & Significance $(\mathrm{p})$ \\
\hline PUVA - Petrolatum & $0.011^{*}$ \\
PUVA - Basis cream & 0.326 \\
PUVA - Glycerine & 0.611 \\
PUVA -Olive oil & 0.799 \\
Petrolatum - Basis cream & $0.043^{*}$ \\
Petrolatum - Glycerine & $0.001^{*}$ \\
Petrolatum -Olive oil & $0.004^{*}$ \\
Basis cream - Glycerine & $0.020^{*}$ \\
Basis cream -Olive oil & 0.118 \\
Olive oil - Glycerine & 0.317 \\
\hline
\end{tabular}

PUVA, psoralen plus ultraviolet A; *, $\mathrm{p}<0.05$ (Wilcoxon signed ranks test) 
enhancing type. ${ }^{1-4}$ So far, despite the studies evaluating the effects of some emollients before UVB therapy in literature, there are relatively few studies demonstrating the effects of emollients immediately before UVA therapy on normal and/or lesional skin. ${ }^{1-12}$ Considering those studies, which vary in regard of the effects of some emollients in UV transmission, it is difficult to predict how any particular preparation may behave in clinical practice. It is obvious that the actual effects will depend on the emollient used, the application dose of the emollient, the type of the application area (normal-appearing or lesional skin), the treatment modality (differences between UVA or UVB wavelengths) as well as the time interval between the application and phototherapy/photochemotherapy. Generally, liquid-based topical agents appear to be more effective on enhancing the UV penetration than thick petrolatum-based compounds. ${ }^{3}$ It was also demonstrated that certain emollients which enhance the UV transmission (e.g., mineral oil, vaseline oil, and glycerol) had a refractive index closest to that of normal stratum corneum. And conversely, the others, which have UV-blocking effects (e.g, petrolatum, coconut oil, and sunflower oil), had a refractive index farthest from that of normal stratum corneum. ${ }^{1}$

In the UVB range, it was shown that a thin $\left(0.1 \mathrm{cc} / 25 \mathrm{~cm}^{2}\right)$ or thick $\left(0.3 \mathrm{cc} / 25 \mathrm{~cm}^{2}\right)$ application of petrolatum can block UVB with the increasing blockage parallel to its thickness. ${ }^{13}$ It was also found that the thick application of petrolatum and white emollient cream resulted in a marked increase in minimal erythema dose although thin applications have negligible effects. However, on the other hand, mineral oil and clear liquid emollient did not significantly affect the transmission of UVB..$^{14}$ In another in vivo study, increases in minimal erythema doses with white petrolatum and basis cream were detected, while no significant effects on UVB transmission were found with glycerine and olive oil..$^{15}$ In addition, Schleider et al. applied various emollients to normal skin and found that vaseline, petrolatum and hydrophilic ointment had the most UVB-blocking effects, whereas liquid emollients such as bath oil and mineral oil had minimal effects. However, no differences were observed when peanut oil and corn oil were applied. ${ }^{16}$

On the basis of the conflicting results with UVB, it should not be an adequate approach to validate the prior results to UVA therapy. It was stated that emollients absorb the shorter wavelengths of UV more strongly than the longer wavelengths. ${ }^{4}$ Kornreich et al., in an in vitro study, found that petrolatum had a lower absorbance value in the UVA (360nm) range compared with the UVB (310nm) range. ${ }^{17}$ In our previous in vivo studies, it was demonstrated that the thin or thick application of petrolatum can block both UVB and UVA transmission; however, it was not possible to compare the results due to the lack of the measurement values. ${ }^{5,13}$ Gabard et al. carried out an in vitro study and they demonstrated that white petrolatum slightly increased the UVA transmission, even though it was not statistically significant. ${ }^{2}$ Similarly, in an in vivo study, Boyvat et al. found a slight but non-significant decrease in the MPD for UVA after the application of white petrolatum. ${ }^{4}$ However, in another study conducted by Birgin et al., it was found that MPD had increased for UVA after the thin or thick application of petrolatum. ${ }^{5}$ Hudson-Peacock et al. carried out an in vitro study demonstrating the photoprotective potential of several emollients as creams, lotions, ointments, emollient bath additives, liquid parafin, glycerine, and coconut oil for UVB and PUVA. They concluded that the emollient oils showed the least photoprotective effects. ${ }^{7}$ Boyvat et al. showed that the application of Urederm hydro (an emollient composed of $10 \%$ urea in oil in-water emulsion) and Decubal (an emollient of the oil in-water type) before the exposure to UVA, had significantly decreased MPD, but the application of Balmandol (a bath oil composed of almond oil, liquid paraffin) had slightly increased the MPD. ${ }^{4}$ In an in vitro study, Gabard et al. demonstrated while the bath oils enhanced the UVA transmission significantly, some emollients containing urea slightly decreased the UVA transmission with no statistically significant change. ${ }^{2}$ In our study, there was an increase in the MPD with petrolatum and basis cream, and a less increase with olive oil. However, only the MPD values detected with petrolatum were found to be statistically significant. Our results, showing the blocking effects of petrolatum on the transmission of UVA, are in accordance with Birgin's results. ${ }^{5}$

We may want to use some emollients before UV therapy to enhance the therapeutic effect in skin diseases. In scaly dermatoses, such as psoriasis, increased scales with multiple air-corneocyte interfaces can increase the reflectance of optic radiations and prevent the complete penetration of therapeutic UV rays. In addition, these plaques with a thicker stratum corneum as compared with healthy skin may prevent a significant amount of therapeutic radiation via the proliferative compartment. ${ }^{1,4}$ Several studies have shown more effective clearing of psoriatic plaques by reducing the reflectance and scattering of light as it transmits through these plaques when various topical emollients are applied in conjunction with phototherapy. ${ }^{12,18-22}$ Berne et al. showed the enhanced response of psoriasis lesions to UVB therapy after the pretreatment with Decubal, a lubricating base of the oil-in-water type..$^{20}$ Abdallah et al., in an in vivo study, demonstrated that the pretreatment of psoriatic plaques with petrolatum may enhance the therapeutic outcome of narrowband-UVB. ${ }^{22}$ In the in vivo study performed by Farr et al., they showed an increase in the light transmission through psoriasis lesions after the application of liquid glycerine. ${ }^{18}$ Penven et al. demonstrated that significantly more plaques were cleared in severe psoriasis on the vaseline oil pre-treated side with UVB therapy. ${ }^{21}$ Jain et al. showed the enhanced response of psoriasis to narrowband-UVB with the pre-irradiation use of mineral oil. ${ }^{19}$ However, George et al. demonstrated that the pre-treatment of plaques with coconut oil showed no significant effect when used before narrowband-UVB and the decreased plaque clearance in the group that received PUVA therapy. ${ }^{12}$ In our study there was a slight decrease in the MPD with glycerine, similar to Farr et al.'s observation. This may potentially aid the therapeutic benefit of phototherapy when glycerine was used prior to UV exposure in psoriasis, leading to shorter exposure times and better dosage adjustment during PUVA therapy, but we must try glycerine on lesional skin to determine its effect. It has long been discussed whether the enhancing effects of emollient pre-treatment on the response to UV therapy occur only in psoriatic skin or also in normal skin. Schleider et al. showed that oils enhance the UV penetration into the psoriatic plaque, while having little or no influence on the UV absorption in normal skin. ${ }^{16}$ However, other studies demonstrated that the application of emollients increases the UV 
transmission in both psoriatic and normal skin. ${ }^{18,23}$ Although the in vitro and/or in vivo studies on normal skin may provide a slight perspective, the precise decision can be made by performing the studies using these emollients on lesional skin. We investigated the effects of lubricant emollients on the UVA transmission based solely on the evaluation of the MPD on normal skin. However, the results obtained by our study may not reflect the effects of emollients on psoriatic or other lesional skin.

\section{CONCLUSIONS}

In conclusion, there is a need for a suitable emollient for pre-irradiation application in skin diseases. It must be taken into consideration that there are a large number of emollients available, so the effects of selected emollients on MPD should be tested before patients are advised to use these before photochemotherapy. According to our findings, the applications of petrolatum and basis cream just before PUVA therapy are not recommended because of their blocking effects. We also observed that glycerine and maybe olive oil can be used before photochemotherapy. Clearly, further clinical studies are needed to clarify the effects of these emollients on the course and outcome of photochemotherapy, and to determine the effects also on lesional skin. $\square$

\section{REFERENCES}

1. Asztalos ML, Heller MM, Lee ES, Koo J.. The impact of emollients on phototherapy: a review. J Am Acad Dermatol. 2013;68:817-24.

2. Gabard B, Treffel P, Bieli E, Schwab S. Emollients and photo(-chemo)therapy: A call for caution. Dermatology. 1996;192:242-5.

3. Otman SG, Edwards C, Pearse AD, Gambles BJ, Anstey AV. Modulation of ultraviolet (UV) transmission by emollients: relevance to narrowband UVB phototherapy and psoralen plus UVA photochemotherapy. Br J Dermatol. 2006;154:963-8.

4. Boyvat A, Erdi H, Birol A, Gurgey E. Interaction of commonly used emollients with photochemotherapy. Photodermatol Photoimmunol Photomed. 2000;16:156-60

5. Birgin B, Fetil E, Illknur T, Tahsin Güneş A, Ozkan S. Effects of topical petrolatum and salicylic acid upon skin photoreaction to UVA. Eur J Dermatol. 2005;15:156-8.

6. Sutton JD. The sunscreening effect of topical sunflower oil. J Am Acad Dermatol. $1983 ; 8: 909-10$.

7. Hudson-Peacock MJ, Diffey BL, Farr PM. Photoprotective action of emollients in ultraviolet therapy of psoriasis. Br J Dermatol. 1994 Mar; 130:361-5.

8. Seité S, Reinhold K, Jaenicke T, Brenden H, Krutmann J, Grether-Beck S. Broadspectrum moisturizer effectively prevents molecular reactions to UVA radiation. Cutis. 2012 Dec;90(6):321-6.

9. Saewan N, Jimtaisong A. Natural products as photoprotection. J Cosmet Dermatol. 2015:14:47-63.

10. Serafini MR, Detoni CB, Menezes Pdos P, Pereira Filho RN, Fortes VS, Vieira MJ, et al. UVA-UVB photoprotective activity of topical formulations containing Morinda citrifolia extract. Biomed Res Int. 2014;2014:587819.

11. Hoffmann K, Kaspar K, Gambichler T, Altmeyer P. Change in ultraviolet (UV) transmission following the application of vaseline to non-irradiated and UVBexposed split skin. Br J Dermatol. 2000;143:532-8.

12. George SA, Bilsland DJ, Wainwright NJ, Ferguson J. Failure of coconut oil to accelerate psoriasis clearance in narrow-band UVB phototherapy or photochemotherapy. Br J Dermatol. 1993;128:301-5.

13. Fetil E, Ozka S, Soyal MC, Ilknur T, Erdem Y, Güneş AT. Effects of topical petrolatum and salicylic acid on the erythemogenicity of UVB. Eur J Dermatol. 2002;12:154-6.

14. Lebwohl M, Martinez J, Weber P, DeLuca R. Effects of topical preparations on the erythemogenicity of UVB: implications for psoriasis phototherapy. J Am Acad Dermatol. 1995;32:469-71.
15. Fetil E, Akarsu S, Ilknur T, Kuşku E, Güneş AT. Effects of some emollients on the transmission of ultraviolet. Photodermatol Photoimmunol Photomed. 2006;22:137-40.

16. Schleider NR, Moskowitz RS, Cort DH, Horwitz SN, Frost P. Effects of emollients on ultraviolet-radiation-induced erythema of the skin. Arch Dermatol. 1979;115:1188-91.

17. Kornreich C, Zheng ZS, Xue GZ, Prystowsky JH. A simple method to predict whether topical agents will interfere with phototerapy. Cutis. 1996;57:113-8.

18. Farr PM, Diffey BL, Steele MC. A preliminary study on the in vivo transmission of light through psoriatic plaques. Photodermatol. 1984;1:87-90.

19. Jain VK, Bansal A, Aggarwal K, Jain K. Enhanced response of childhood psoriasis to narrow-band UV-B phototherapy with preirradiation use of mineral oil. Pediatr Dermatol. 2008:25:559-64.

20. Berne B, Blom I, Spångberg S. Enhanced response of psoriasis to UVB therapy after pretreatment with a lubricating base. A single-blind controlled study. Acta Derm Venereol. 1990;70:474-7.

21. Penven K, Leroy D, Verneuil L, Faguer K, Dompmartin A. Evaluation of vaseline oil applied prior to UVB TL01 phototherapy in the treatment of psoriasis. Photodermatol Photoimmunol Photomed. 2005;21:138-41.

22. Abdallah MA, El-Khateeb EA, Abdel-Rahman SH. The influence of psoriatic plaques pretreatment with crude coal tar vs. petrolatum on the efficacy of narrowband ultraviolet B: a half-vs.-half intra-individual double-blinded comparative study. Photodermatol Photoimmunol Photomed. 2011;27:226-30.

23. Leroy D, Dompmartin A, Deschamps P. Increased penetration of epidermis by high intensity ultraviolet rays following the application of Vaseline oil. Photodermatol. 1986;3:51-2.

How to cite this article: Akarsu S, Fetil E, Ozbagcivan O, Gunes AT. Blocking or enhancing effects of some basic emollients in UVA penetration. An Bras Dermatol. 2018;93(2):238-41. 